\title{
The Least-Energy Sign-Changing Solutions for Planar Schrödinger-Newton System with an Exponential Critical Growth
}

\author{
Wenbo Wang ${ }^{1}$, Wei Zhang ${ }^{2}$, Yongkun $\mathrm{Li}^{1, *}$ \\ ${ }^{1}$ School of Mathematics and Statistics, Yunnan University, Kunming, Yunnan, P.R. China \\ ${ }^{2}$ School of Statistics and Mathematics, Yunnan University of Finance and Economics, Kunming, Yunnan, P.R. China
}

Email address:

yklie@ynu.edu.cn (Yongkun Li)

*Corresponding author

To cite this article:

Wenbo Wang, Wei Zhang, Yongkun Li. The Least-Energy Sign-Changing Solutions for Planar Schrödinger-Newton System with an Exponential Critical Growth. Pure and Applied Mathematics Journal. Vol. 9, No. 6, 2020, pp. 118-123.

doi: $10.11648 /$ j.pamj.20200906.13

Received: February 8, 2020; Accepted: September 8, 2020; Published: September 29, 2020

\begin{abstract}
In 1990, the notion of critical growth in $\mathbb{R}^{2}$ was introduced by Adimurthi and Yadava. Soon afterwards, de Figueiredo, Miyagaki and Ruf also studied the solvability of elliptic equations in dimension two. They treated the problems in the subcritical and the critical case. In 2019, Alves and Figueieredo proved the existence of a positive solution for a planar Schrodinger-Poisson system, where the nonlinearity is a continuous function with exponent critical growth. Also, in 2020, Chen and Tang investigated the planar Schrodinger-Poisson system with the critical growth nonlinearity. Under the axially symmetric assumptions, they obtained infinitely many pairs solutions and ground states. In this work, motivated by the works mentioned above and Z. Angew. Math. Phys., 66 (2015) 3267-3282, Z. Angew. Math. Phys., 67 (2016) 102, 18, we study the planar Schrödinger-Newton system with a Coulomb potential where the nonlinearity $f$ is autonomous nonlinearity which belongs to $C^{1}$ and satisfies super-linear at zero and exponential critical at infinity. Moreover, we need that $f$ satisfies the Nehari type monotonic condition. We obtain a least-energy sign-changing solution via the variational method. To be more precise, we define the sign-changing Nehari manifold. And the least-energy sign-changing solution is obtained by minimizing the energy functional on the sign-changing Nehari manifold.
\end{abstract}

Keywords: Schrödinger-Newton System, The Exponential Critical Growth, Sign-Changing Solutions,

Sign-Changing Nehari Manifold

\section{Introduction and Main Results}

In the present paper, we are concerned with the following Schrödinger-Newton system with a Coulomb potential

$$
\left\{\begin{array}{l}
-\Delta u+u+2 \pi \phi u+\int_{\mathbb{R}^{2}} \frac{[u(y)]^{2}}{|x-y|} d y u=f(u), \text { in } \mathbb{R}^{d}, \\
\Delta \phi=u^{2}, \text { in } \mathbb{R}^{d} .
\end{array}\right.
$$

Problems of the type (1) arise in many problems from physics. And we refer the readers to [17], where (1) appears in a quantum mechanical context in the case $d \leq 3$. For the case $d=3,(1)$ is called the Schrödinger-Poisson system and it has been well studied, see for example $[3,5,16,20,22]$ and the references therein. However, much less is known about the case $d=2$.

Motivated by the papers $[2,10,12,15,19]$, the purpose of this paper is to study the existence of least-energy sign-changing solutions of the planar problem (1) with an exponential critical growth (see [2]). We mention that this notion of criticality was introduced by Adimurthi and Yadava [1], see also de Figueiredo, Miyagaki and Ruf [13]. We refer the readers to $[6,7,8,9,11]$ for related problems and for recent advances on planar Schrödinger-Newton system. In order to state our main result, we assume that $\left(f_{1}\right) f \in C^{1}(\mathbb{R}, \mathbb{R})$ and $f(u)=o(|u|)$ as $u \rightarrow 0$; 
$\left(f_{2}\right)$ There exists an $\alpha_{0}>0$ such that

$$
\begin{aligned}
& \lim _{|u| \rightarrow \infty} \frac{f(u)}{\exp \left(\alpha u^{2}\right)}=0 \text { for } \alpha>\alpha_{0}, \\
& \lim _{|u| \rightarrow \infty} \frac{f(u)}{\exp \left(\alpha u^{2}\right)}=+\infty \text { for } \alpha<\alpha_{0}
\end{aligned}
$$

$\left(f_{3}\right) \frac{f(u)}{|u|^{3}}$ is a strictly increasing function of $u \in \mathbb{R} \backslash\{0\}$;

$\left(f_{4}\right)$ There is a $q>4$ such that $F(u) \geq \frac{1}{q}|u|^{q}$, where $F(u)=\int_{0}^{u} f(t) d t$.

Remark 1.1. $f(u)=u|u|^{5} \exp \left(\frac{\pi-1}{m} u^{2}\right)$ satisfies the assumptions $\left(f_{1}\right)-\left(f_{4}\right)$.

Now we state our first result as follows.

Theorem 1.1. For $d=2$, suppose that $\left(f_{1}\right)-\left(f_{4}\right)$ are satisfied. Then for any $\alpha \in\left(0, \frac{\pi}{m}\right)$, where $m>0$ will be defined in (20), (1) possesses a least-energy sign-changing solution.

Remark 1.2. We point out that we can define $m$ without using the condition $\alpha \in\left(0, \frac{\pi}{m}\right)$. It seems that the condition $\alpha \in\left(0, \frac{\pi}{m}\right)$ is fussy but it is used to prove the minimizing sequence of $m$ is bounded, and please see Lemma 3.1. Up to now, we have not been able to remove it..

The paper is organized as follows. In Section 2 , let $\left(f_{1}\right)-$ $\left(f_{4}\right)$ be satisfied, we are going to establish the variational setting and give some preliminaries. In Section 3, with the additional condition $\alpha \in\left(0, \frac{\pi}{m}\right)$, we are devoted to show that $m$ is achieved and the minimizer is a critical point. The section 4 is the conclusion, we summarize our main results and the main idea of the proof.

\section{Variational Setting and Preliminaries}

We formally formulate problem (1) in a variational way as

$$
I(u)=\frac{1}{2}\|u\|_{H^{1}}^{2}+\frac{1}{4} \int_{\mathbb{R}^{2}}\left(\ln (|x|) * u^{2}\right) u^{2} d x+\frac{1}{4} \int_{\mathbb{R}^{2}}\left(|x|^{-1} * u^{2}\right) u^{2} d x-\int_{\mathbb{R}^{2}} F(u) d x, u \in X,
$$

where

$$
\ln (|\cdot|) * u^{2}(x)=\int_{\mathbb{R}^{2}} \ln (|x-y|) u^{2}(y) d y,|\cdot|^{-1} * u^{2}(x)=\int_{\mathbb{R}^{2}} \frac{u^{2}(y)}{|x-y|} d y,
$$

and $X$ is defined as tha in [2].

Using $\left(f_{1}\right)-\left(f_{2}\right)$, for each $\varepsilon>0$ and $\alpha>\alpha_{0}$, we have

$$
|F(u)| \leq \varepsilon|u|^{2}+C(\varepsilon)|u|^{p}\left[\exp \left(\alpha|u|^{2}\right)-1\right],
$$

where $p>2$. At first, according to [2, 4, 14], we give the following lemma which is used to estimate the nonlinearity.

Lemma 2.1. If $\alpha>0$ and $u \in H^{1}\left(\mathbb{R}^{2}\right)$, then $\exp \left(\alpha u^{2}\right)-1 \in L^{1}\left(\mathbb{R}^{2}\right)$. Moreover, if $\|\nabla u\|_{L^{2}} \leq 1,\|u\|_{L^{2}} \leq M$ and $\alpha<4 \pi$, then there exists $C>0$ independent of $u$ such that

$$
\int_{\mathbb{R}^{2}}\left[\exp \left(\alpha u^{2}\right)-1\right] d x \leq C .
$$

Similar to $[2,12]$, in view of Lemma 2.1 , it is easy to check that the functional $I$ belongs to $C^{1}(X, \mathbb{R})$. Define constraint:

$$
M=\left\{u \in X: u^{ \pm} \neq 0,\left\langle I^{\prime}(u), u^{+}\right\rangle=\left\langle I^{\prime}(u), u^{-}\right\rangle=0\right\} .
$$

Lemma 2.2. For $u \in E$ with $u^{ \pm} \neq 0$, then there exists a unique pair $\left(s_{u}, t_{u}\right)$ of positive numbers such that $s_{u} u^{+}+t_{u} u^{-} \in M$. Moreover, if $s, t \geq 0$ and $(s, t) \neq\left(s_{u}, t_{u}\right)$, we have

$$
I\left(s u^{+}+t u^{-}\right)<I\left(s_{u} u^{+}+t_{u} u^{-}\right) .
$$

Proof. For $u \in E$ with $u^{ \pm} \neq 0$, similar to [18], we define

$$
\begin{aligned}
g(\mu, s, t):= & s^{2}\left\|u^{+}\right\|_{H^{1}}^{2}+s^{4} \int_{\mathbb{R}^{2}}\left\{\left[\left(\ln |x| *\left|u^{+}\right|^{2}\right)+\left(|x|^{-1} *\left|u^{+}\right|^{2}\right)\right]\left|u^{+}\right|^{2}\right\} d x \\
& \left.+\mu s^{2} t^{2} \int_{\mathbb{R}^{2}}\left\{\left[\left(\ln |x| *\left|u^{-}\right|^{2}\right)+|x|^{-1} *\left|u^{-}\right|^{2}\right)\right]\left|u^{+}\right|^{2}\right\} d x-\int_{\mathbb{R}^{3}} f\left(s u^{+}\right) s u^{+} d x,
\end{aligned}
$$

and

$$
\begin{aligned}
h(\mu, s, t):= & t^{2}\left\|u^{-}\right\|_{H^{1}}^{2}+t^{4} \int_{\mathbb{R}^{2}}\left\{\left[\left(\ln |x| *\left|u^{-}\right|^{2}\right)+\left(|x|^{-1} *\left|u^{-}\right|^{2}\right)\right]\left|u^{-}\right|^{2}\right\} d x \\
& \left.+\mu s^{2} t^{2} \int_{\mathbb{R}^{2}}\left\{\left[\left(\ln |x| *\left|u^{+}\right|^{2}\right)+|x|^{-1} *\left|u^{+}\right|^{2}\right)\right]\left|u^{-}\right|^{2}\right\} d x-\int_{\mathbb{R}^{3}} f\left(t u^{-}\right) t u^{-} d x .
\end{aligned}
$$


Consider the solvability of the following system

$$
\left\{\begin{array}{l}
g(\mu, s, t)=0 \\
h(\mu, s, t)=0
\end{array}\right.
$$

Define $\mathcal{Z}:=\{\zeta: 0 \leq \zeta \leq 1$ such that (10) is uniquely solvable in $\left.\left(\mathbb{R}_{+}, \mathbb{R}_{+}\right)\right\}$, where $\mathbb{R}_{+}=(0, \infty)$. Obviously, $g(0, s, t)$ is independent of $t$ and $h(0, s, t)$ is independent of $s$. By $\left(f_{1}\right)$ and $\left(f_{2}\right)$, noting that

$$
\begin{aligned}
& \ln (r)=\ln (1+r)-\ln \left(1+\frac{1}{r}\right), \\
& 0 \leq \ln \left(1+\frac{1}{r}\right) \leq \frac{1}{r}, \text { for } r>0,
\end{aligned}
$$

Thus,

$$
\begin{aligned}
\left.\operatorname{frac} \partial g(\mu, s, t) \partial s\right|_{\left(\mu_{0}, \bar{s}, \bar{t}\right)} & =\bar{s}\left\|u^{+}\right\|_{H^{1}}^{2}+3 \bar{s}^{3} \int_{\mathbb{R}^{2}}\left\{\left[\left(\ln |x| *\left|u^{+}\right|^{2}\right)+\left(|x|^{-1} *\left|u^{+}\right|^{2}\right)\right]\left|u^{+}\right|^{2}\right\} d x \\
& \left.+\mu_{0} \bar{s} \bar{t}^{2} \int_{\mathbb{R}^{2}}\left\{\left[\left(\ln |x| *\left|u^{-}\right|^{2}\right)+|x|^{-1} *\left|u^{-}\right|^{2}\right)\right]\left|u^{+}\right|^{2}\right\} d x-\int_{\mathbb{R}^{3}} f^{\prime}\left(\bar{s} u^{+}\right) \bar{s}\left|u^{+}\right|^{2} d x \\
& \left.<-2 \bar{s}\left\|u^{+}\right\|_{H^{1}}^{2}-2 \mu_{0} \bar{s} \bar{t}^{2} \int_{\mathbb{R}^{2}}\left\{\left[\left(\ln |x| *\left|u^{-}\right|^{2}\right)+|x|^{-1} *\left|u^{-}\right|^{2}\right)\right]\left|u^{+}\right|^{2}\right\} d x
\end{aligned}
$$

In view of (11), we have

$$
\left.\left.\frac{\partial g(\mu, s, t)}{\partial s}\right|_{\left(\mu_{0}, \bar{s}, \bar{t}\right)}<-2 \bar{s}\left\|u^{+}\right\|_{H^{1}}^{2}-2 \mu_{0} \bar{s} \bar{t}^{2} \int_{\mathbb{R}^{2}}\left[\ln (1+|x|) *\left|u^{-}\right|^{2}\right)\right]\left|u^{+}\right|^{2} d x<0 .
$$

and

$$
\left.\left.\frac{\partial g(\mu, s, t)}{\partial t}\right|_{\left(\mu_{0}, \bar{s}, \bar{t}\right)}=2 \mu_{0} \bar{s}^{2} \bar{t} \int_{\mathbb{R}^{2}}\left\{\left[\left(\ln |x| *\left|u^{-}\right|^{2}\right)+|x|^{-1} *\left|u^{-}\right|^{2}\right)\right]\left|u^{+}\right|^{2}\right\} d x .
$$

And for $h(\mu, s, t)$, we have similar results. Therefore, it yields that

$$
\operatorname{det}\left(\begin{array}{ll}
\left.\frac{\partial g(\mu, s, t)}{\partial s}\right|_{\left(\mu_{0}, \bar{s}, \bar{t}\right)} & \left.\frac{\partial g(\mu, s, t)}{\partial t}\right|_{\left(\mu_{0}, \bar{s}, \bar{t}\right)} \\
\left.\frac{\partial h(\mu, s, t)}{\partial s}\right|_{\left(\mu_{0}, \bar{s}, \bar{t}\right)} & \left.\frac{\partial h(\mu, s, t)}{\partial t}\right|_{\left(\mu_{0}, \bar{s}, \bar{t}\right)}
\end{array}\right)>0
$$

Jointly with the implicit function theorem, we can obtain the desired conclusion. We deduce that $I_{\mu}\left(s u^{+}+t u^{-}\right) \rightarrow$ $-\infty$ uniformly as $|(s, t)| \rightarrow \infty$. Up to this stage, it is sufficient to check that a maximum point cannot be achieved on $\left(0 \times \mathbb{R}_{+}\right) \cup\left(\mathbb{R}_{+} \times 0\right)$. Otherwise, if $(0, \bar{t})$ is a maximum point. In view of (11), for $s>0$ small enough, with [2, Lemma 2.5] in hand, it holds that

$$
\frac{\partial I\left(s u^{+}+\bar{t} u^{-}\right)}{\partial s} \geq 2 s C_{1}+s^{3} C_{2}+s C_{3}-\varepsilon s C_{4}-C(\varepsilon) s^{p} C_{5}>0,
$$

which is a contradiction.

Since $u \in M$, by $\left(f_{3}\right), f(u) u-4 F(u) \geq 0$, one has

$$
I(u)=I(u)-\frac{1}{4}\left\langle I^{\prime}(u), u\right\rangle \geq 0 .
$$

We can define the minimization problem

$$
m:=\inf _{u \in M} I(u) .
$$

\section{The Proof of Theorem 1.1}

Lemma 3.1. $m>0$ can be achieved.

Proof. Let $\left\{u_{n}\right\} \subset M$ be a minimizing sequence. It follows that

$$
m+o_{n}(1)=I\left(u_{n}\right)-\frac{1}{4}\left\langle I^{\prime}\left(u_{n}\right), u\right\rangle \geq \frac{1}{4}\left\|u_{n}\right\|_{H^{1}}^{2},
$$

which implies that $\left\{u_{n}\right\}$ is bounded in $H^{1}\left(\mathbb{R}^{2}\right)$. We can assume that $u_{n}^{ \pm} \rightarrow u_{0}^{ \pm}$in $H^{1}\left(\mathbb{R}^{2}\right)$. Since $u_{n} \in M$, and

$$
\int_{\mathbb{R}^{2}}\left(\ln |x| * u_{n}^{2}+|x|^{-1} * u_{n}^{2}\right)\left|u_{n}^{ \pm}\right|^{2} d x \geq 0
$$

one has

$$
\left\|u_{n}^{ \pm}\right\|_{H^{1}}^{2} \leq \int_{\mathbb{R}^{2}} f\left(u_{n}^{ \pm}\right) u_{n}^{ \pm} d x \leq \varepsilon \int_{\mathbb{R}^{2}}\left|u_{n}^{ \pm}\right|^{2} d x+C(\varepsilon) \int_{\mathbb{R}^{2}}\left|u_{n}^{ \pm}\right|^{p}\left[\exp \left(\alpha\left|u_{n}^{ \pm}\right|^{2}\right)-1\right] d x
$$


If $\left\|u_{n}^{ \pm}\right\|_{H^{1}} \rightarrow 0$, using Hölder inequality with $s>1$ and $s \approx 1$, it holds that

$$
\left.\int_{\mathbb{R}^{2}}\left|u_{n}^{ \pm}\right|^{p}\left[\mid \exp \left(\alpha\left|u_{n}^{ \pm}\right|^{2}\right)-1\right]\right) d x \leq\left(\int_{\mathbb{R}^{2}}\left|u_{n}^{ \pm}\right|^{s^{\prime} p} d x\right)^{\frac{1}{s^{\prime}}}\left(\int_{\mathbb{R}^{2}}\left[\exp \left(\alpha s\left\|u_{n}^{ \pm}\right\|_{H^{1}}^{2}\left(\frac{u_{n}^{ \pm}}{\left\|u_{n}^{ \pm}\right\|_{H^{1}}}\right)^{2}\right)-1\right] d x\right)^{\frac{1}{s}} .
$$

By $\left\|u_{n}^{ \pm}\right\|_{H^{1}} \rightarrow 0$, for $n$ large enough, it holds that

$$
\alpha s\left\|u_{n}^{ \pm}\right\|_{H^{1}}^{2}<4 \pi
$$

By Lemma 2.1 and Sobolev embedding, we get

$$
(1-C \varepsilon)\left\|u_{n}^{ \pm}\right\|_{H^{1}}^{2} \leq C C(\varepsilon)\left\|u_{n}^{ \pm}\right\|_{H^{1}}^{p} .
$$

It is absurd. So $m>0$. And we claim that there are $R, \eta>0$ such that

$$
\liminf _{n \rightarrow \infty} \int_{B_{R}\left(y_{n}\right)}\left|u_{n}^{ \pm}\right|^{2} d x \geq \eta
$$

If it is false, using Lion's lemma, we get $u_{n}^{ \pm} \rightarrow 0$ in $L^{t}\left(\mathbb{R}^{2}\right)$ for all $t \in[2, \infty)$. Noting (21) and taking into account $\alpha \in\left(0, \frac{\pi}{m}\right)$, it yields that

$$
\alpha s\left\|u_{n}^{ \pm}\right\|_{H^{1}}^{2}<4 \pi
$$

So we have

$$
\left\|u_{n}^{ \pm}\right\|_{H^{1}}^{2} \leq \int_{\mathbb{R}^{2}} f\left(u_{n}^{ \pm}\right) u_{n}^{ \pm} d x \leq \varepsilon \int_{\mathbb{R}^{2}}\left|u_{n}^{ \pm}\right|^{2} d x+C(\varepsilon) C\left(\int_{\mathbb{R}^{2}}\left|u_{n}^{ \pm}\right|^{s^{\prime} p} d x\right)^{\frac{1}{s^{\prime}}} \rightarrow 0,
$$

which is a contradiction. Thus, $u_{0}^{ \pm} \neq 0$. As our problem is invariant by translations, according to [2, Corollary 4.4], up to a translation, we have that $\left\{u_{n}\right\}$ is bounded in $X$. Furthermore, it gives that

$$
\int_{\mathbb{R}^{2}} f\left(u_{n}^{ \pm}\right) u_{n}^{ \pm} d x=\int_{\mathbb{R}^{2}} f\left(u_{0}^{ \pm}\right) u_{0}^{ \pm} d x+o_{n}(1)
$$

and

$$
\int_{\mathbb{R}^{2}} F\left(u_{n}^{ \pm}\right) d x=\int_{\mathbb{R}^{2}} F\left(u_{0}^{ \pm}\right) d x+o_{n}(1) .
$$

By Lemma 2.2, there exists $(s, t) \in(0, \infty) \times(0, \infty)$ such that $s u_{0}^{+}+t u_{0}^{-} \in M$. Moreover, by Fatou's Lemma, combining with (11), we get

$$
\int_{\mathbb{R}^{2}}\left\{\left[\left(\ln |x| *\left|u_{0}\right|^{2}\right)+\left(|x|^{-1} *\left|u_{0}\right|^{2}\right)\right]\left|u_{0}^{ \pm}\right|^{2}\right\} d x \leq \liminf _{n \rightarrow \infty} \int_{\mathbb{R}^{2}}\left\{\left[\left(\ln |x| *\left|u_{n}\right|^{2}\right)+\left(|x|^{-1} *\left|u_{n}\right|^{2}\right)\right]\left|u_{n}^{ \pm}\right|^{2}\right\} d x .
$$

Thus, it follows that

$$
\left\|u_{0}^{ \pm}\right\|_{H^{1}}^{2} \leq \int_{\mathbb{R}^{2}} f\left(u_{0}^{ \pm}\right) u_{0}^{ \pm} d x \text { and } s^{2}\left\|u_{0}^{ \pm}\right\|_{H^{1}}^{2} \leq \int_{\mathbb{R}^{2}} f\left(s u_{0}^{ \pm}\right) s u_{0}^{ \pm} d x .
$$

Therefore, we get $0<s \leq 1$. Similarly, $0<t \leq 1$. By $\left(f_{3}\right)$, we have

$$
u f(u)-4 F(u)
$$

is a nonnegative function, increasing in $|u|$. So we have

$$
\begin{aligned}
m & \leq I\left(s u_{0}^{+}+t u_{0}^{-}\right)-\frac{1}{4}\left\langle I^{\prime}\left(s u_{0}^{+}+t u_{0}^{-}\right), s u_{0}^{+}+t u_{0}^{-}\right\rangle \\
& \leq \frac{1}{4}\left\|u_{0}\right\|_{H^{1}}^{2}+\frac{1}{4} \int_{\mathbb{R}^{2}}\left[u_{0} f(u)-4 F\left(u_{0}\right)\right] d x \\
& \leq \liminf _{n \rightarrow \infty}\left[I\left(u_{n}\right)-\frac{1}{4}\left\langle I^{\prime}\left(u_{n}\right), u_{n}\right\rangle\right]=m .
\end{aligned}
$$

The claim is done.

Lemma 3.2. Suppose that $u \in M$ and $I(u)=m$, then $u$ is a sign-changing critical point of $I$.

Proof. Assuming the contrary that $u$ is not a critical point of $I$. We can find $\psi \in C_{0}^{\infty}\left(\mathbb{R}^{2}\right)$ satisfying $\psi^{ \pm} \not \equiv 0$ such that 
$\left.\left\langle I^{\prime}(u), \psi\right)\right\rangle \leq-1$. Choose $\varepsilon>0$ very small such that

$$
\left\langle I^{\prime}\left(s u^{+}+t u^{-}+\sigma \psi\right), \psi\right\rangle \leq-\frac{1}{2}, \forall(s, t, \sigma) \in B_{\varepsilon}(1,1,0),
$$

where $B_{\varepsilon}(1,1,0)$ denotes the ball of radius $\varepsilon$ centered at $(1,1,0)$. And introduce a cut-off function $0 \leq \eta \leq 1$ such that $\eta(s, t)=1$ for $(s, t) \in \overline{B_{\frac{\varepsilon}{2}}(1,1)}$ and $\eta(s, t)=0$ for $(s, t) \in B_{\varepsilon}^{c}(1,1)$. We perturb the original curve $s u^{+}+t u^{-}$, for $s, t \geq 0$,

$$
\gamma(s, t)=\left\{\begin{array}{l}
s u^{+}+t u^{-}, \quad \text { if }(s, t) \in B_{\varepsilon}^{c}(1,1) \\
s u^{+}+t u^{-}+\varepsilon \eta(s, t) \psi, \text { if }(s, t) \in B_{\varepsilon}(1,1) .
\end{array}\right.
$$

Noting that $\gamma(s, t)$ is a continuous curve in $(X,\|\cdot\|)$, and eventually choosing a smaller $\varepsilon$, we obtain that $\gamma(s, t)^{ \pm} \not \equiv 0$ for $(s, t) \in B_{\varepsilon}(1,1)$. We have the following claim.

Claim 3.1.

$$
\sup _{s, t \geq 0} I(\gamma(s, t))<m
$$

If $(s, t) \in B_{\varepsilon}^{c}(1,1)$, according to Lemma 2.2, $I(\gamma(s, t))<m$. If $(s, t) \in B_{\varepsilon}(1,1)$, by using the mean value theorem, we can find a suitable $\bar{\sigma} \in(0, \varepsilon)$ such that

$$
\begin{aligned}
\gamma(s, t) & =\left(s u^{+}+t u^{-}\right)+\left\langle I^{\prime}\left(s u^{+}+t u^{-}+\bar{\sigma} \eta(s, t) \psi\right), \eta(s, t) \psi\right\rangle \\
& \leq I\left(s u^{+}+t u^{-}\right)-\frac{1}{2} \eta(s, t) \\
& <m,
\end{aligned}
$$

Where in the first inequality we have used (36).

However, observing that Lemma 2.2, it holds that for $(s, t) \in\left(1-\frac{\varepsilon}{2}, 1\right) \times\left(1-\frac{\varepsilon}{2}, 1\right)$,

$$
\left\langle I^{\prime}\left(s u^{+}+t u^{-}+\varepsilon \psi\right), u^{+}\right\rangle>0
$$

and

$$
\left\langle I^{\prime}\left(s u^{+}+t u^{-}+\varepsilon \psi\right), u^{-}\right\rangle>0 .
$$

Similarly, for $(s, t) \in\left(1,1+\frac{\varepsilon}{2}\right) \times\left(1,1+\frac{\varepsilon}{2}\right)$, we have

$$
\left\langle I^{\prime}\left(s u^{+}+t u^{-}+\varepsilon \psi\right), u^{+}\right\rangle<0
$$

and

$$
\left\langle I^{\prime}\left(s u^{+}+t u^{-}+\varepsilon \psi\right), u^{-}\right\rangle<0 .
$$

Thus, there exists a unique pair $\left(s_{0}, t_{0}\right) \in\left(1-\frac{\varepsilon}{2}, 1+\frac{\varepsilon}{2}\right) \times$ $\left(1-\frac{\varepsilon}{2}, 1+\frac{\varepsilon}{2}\right)$ such that

$$
s_{0} u^{+}+t_{0} u^{-}+\varepsilon \eta\left(s_{0, t_{0}}\right) \psi \in M,
$$

which contradicts the claim.

\section{Conclusions}

In this research, under the exponential critical growth condition $\left(f_{2}\right)$ and other suitable conditions, we prove that the planar Schrödinger-Newton system with a Coulomb potential admits a least-energy sign-changing solution. Our basic leitmotiv is to seek the minimizer of the energy functional on the sign-changing Nehari manifold. So, at first, we show the sign-changing Nehari manifold (see (6)) is well defined. Furthermore, we show that we can define the minimization problem. In addition, we prove that minimizer is achieved. Finally, we show that the minimizer is a critical point. Clearly, the minimizer is sign-changing. Also, a example satisfying our assumptions is given in our research.

\section{Acknowledgements}

This work is supported by the National Natural Science Foundation of China $(11901514,11861072)$ and the project of scientific research foundation of Yunnan University of Finance and Economics (2019B02) and Postdoctoral Directional Training of Yunnan Province.

\section{References}

[1] Adimurthi, S. L. Yadava, Multiplicity results for semilinear elliptic equations in bounded domian of $\mathbb{R}^{2}$ involving critical exponents, Ann. Sc. Norm. Super. Pisa, CI. Sci. 17 (1990) 481-504.

[2] C. O. Alves, G. M. Figueieredo, Existence of positive solution for a planar Schrödinger-Poisson system with exponential growth, J. Math. Phys., 60 (2019) 011503.

[3] V. Benci, D. Fortunato, An eigenvalue problem for the Schrödinger-Maxwell equations, Topol. Methods Nonlinear Anal., 11 (1998) 283-293.

[4] D. Cao, Nontrivial solutions of semilinear elliptic equation with critical exponent in $\mathbb{R}^{2}$, Comm. Partial Differential Equations, 17 (1992) 407-435.

[5] S. Chen, X. Tang, Ground state sign-changing solutions for a class of Schrödinger-Poisson system type problems in $\mathbb{R}^{3}$, Z. Angew. Math. Phys.,67 (2016) 102, 18 pp. 
[6] S. Chen, J. Shi, X. Tang, Ground state solutions of Nehari-Pohozaev type for the planar Schrödinger-Poisson system with general nonlinearity, Discrete Contin. Dyn. Syst., 39 (2019) 5867-5889.

[7] S. Chen, X. Tang, Existence of ground state solutions for the planar axially symmetric Schrödinger-Poisson system, Discrete Contin. Dyn. Syst. Ser. B, 24 (9)(2019) 4685-4702.

[8] S. Chen, X. Tang, On the planar SchrödingerPoisson system with the axially symmetric potential, J. Differential Equations, 268 (2020) 945-976.

[9] S. Chen, X. Tang, Axially symmetric solutions for the planar Schrödinger-Poisson system with critical exponential growth, J. Differential Equations, 269 (2020) 9144-9174.

[10] P. Choquard, J. Stubbe, M. Vuffray,Stationary solutions of the Schrödinger Newton model an ODE approach, Differ. Integral Equ., 21 (2008) 665-679.

[11] S. Cingolani and L. Jeanjean, Stationary waves with prescribed $L^{2}$-norm for the planar Schrödinger-Poisson system, SIAM J. Math. Anal., 51 (2019) 3533-3568.

[12] S. Cingolani, T. Weth, On the Schrödinger-Poisson system, Ann. Inst. H. Poincaré Anal. Non Linéaire, 33 (2016) 169-197.

[13] D.G. de Figueiredo, O.H. Miyagaki, B. Ruf, Elliptic equations in $\mathbb{R}^{2}$ with nonlinearities in the critical growth range, Calc. Var. Partial Differential Equations, 3 (1995) 139-153.
[14] J. M. Bezerra Do ó, N-Laplacian equations in $\mathbb{R}^{N}$ with critical growth, Abstr. Appl. Anal., 2 (1997) 301-315.

[15] M. Du, T. Weth, Ground states and high energy solutions of the planar Schrödinger-Poisson system, Nonlinearity, 30 (2017) 3492-3515.

[16] X. He, W. Zou, Existence and concentration of ground states for Schrödinger-Poisson equations with critical growth, J. Math. Phys., 53 (2012) 143-162.

[17] N. J. Mauser, The Schrödinger-Poisson- $X_{\alpha}$ equation, Appl. Math. Lett., 14 (2001) 759-763.

[18] W. Shuai, Q. Wang, Existence and asymptotic behavior of sign-changing solutions for the nonlinear SchrödingerPoisson system in $\mathbb{R}^{3}$, Z. Angew. Math. Phys., 66 (2015) 3267-3282.

[19] J. Stubbe, Bound states of two-dementional SchrödingerNewton equation, arXiv: 0807. 4059v1. 2008.

[20] Z. Wang, H. Zhou, Sign-changing solutions for the nonlinear Schrödinger-Poisson system in $\mathbb{R}^{3}$, Calc. Var. Partial Differential Equations, 52 (2015) 927-943.

[21] L. Wen, S. Chen, V. D. Radulescu, Axially symmetric solutions of the Schrödinger-Poisson system with zero mass potential in $\mathbb{R}^{2}$, Appl. Math. Lett., 104 (2020) 106244.

[22] L. Zhao, F. Zhao, Positive solutions for SchrödingerPoisson equations with critical exponent, Nonlinear Anal., 70 (2009) 2150-2164. 